\title{
Spatial distribution of ions in a linear octopole radio-frequency ion trap in the space-charge limit
}

\author{
Takuya Majima, ${ }^{*}$ Gabriele Santambrogio, ${ }^{\dagger}$ and Christof Bartels ${ }^{\ddagger}$ \\ East Tokyo Laboratory, Genesis Research Institute, Inc., 717-86 Futamata, Ichikawa, Chiba 272-0001, Japan \\ Akira Terasaki ${ }^{\S}$ and Tamotsu Kondow" \\ Cluster Research Laboratory, Toyota Technological Institute in East Tokyo Laboratory, Genesis Research Institute, \\ Inc., 717-86 Futamata, Ichikawa, Chiba 272-0001, Japan \\ Jan Meinen and Thomas Leisner \\ Institute for Meteorology and Climate Research, Karlsruhe Institute of Technology (KIT), 76131 Karlsruhe, Germany
}

(Received 23 January 2012; published 15 May 2012)

\begin{abstract}
We have explored the spatial distribution of an ion cloud trapped in a linear octopole radio-frequency (rf) ion trap. The two-dimensional distribution of the column density of stored $\mathrm{Ag}_{2}^{+}$was measured via photofragment-ion yields as a function of the position of the incident laser beam over the transverse cross section of the trap. The profile of the ion distribution was found to be dependent on the number of loaded ions. Under high ion-loading conditions with a significant space-charge effect, ions form a ring profile with a maximum at the outer region of the trap, whereas they are localized near the center axis region at low loading of the ions. These results are explained quantitatively by a model calculation based on equilibrium between the space-charge-induced potential and the effective potential of the multipole rf field. The maximum adiabaticity parameter $\eta_{\max }$ is estimated to be about 0.13 for the high ion-density condition in the present octopole ion trap, which is lower than typical values reported for low ion densities; this is probably due to additional instability caused by the space charge.
\end{abstract}

DOI: 10.1103/PhysRevA.85.053414

PACS number(s): 37.10.Ty, 41.90.+e, 36.40.Mr

\section{INTRODUCTION}

Linear multipole radio-frequency (rf) ion traps have become increasingly important in atomic, molecular, and cluster physics [1,2]. In spectroscopic studies, for example, these traps improve the resolution by enabling cooling with a neutral buffer gas and improve the sensitivity by enabling accumulation and, thereby, increasing the number density [3-14]. In gas-phase reactivity studies, multipole rf traps have proven ideal in thermalizing all degrees of freedom of reagents and in providing accurate knowledge of their concentrations [15-17]. The success of these traps is due to the characteristics of the time-averaged potential experienced by the trapped ions, the effective potential $\Phi_{\text {eff }}$. The strength of the effective potential is proportional to $p^{2}$ for a $2 p$-pole trap, and its dependence on the distance $r$ from the central axis is proportional to $r^{2 p-2}$ [18]. For high values of $p$, traps are particularly deep and have a large nearly field-free region around the axis, which guarantees little rf heating [18-21]. The deeper a trap, i.e., the larger the phase space acceptance, the larger the fraction of ions that can be captured and accumulated. Additionally,

*Present address: Quantum Science and Engineering Center, Kyoto University, Gokasho, Uji, Kyoto 611-0011, Japan.

${ }^{\dagger}$ Present address: Fritz-Haber-Institut der Max-PlanckGesellschaft, Faradayweg 4-6, 14195 Berlin, Germany.

${ }^{\ddagger}$ Present address: Department of Dynamics at Surfaces, Max Planck Institute for Biophysical Chemistry, Am Faßberg 11, D-37077 Göttingen, Germany.

§Present address: Department of Chemistry, Kyushu University, 6-10-1 Hakozaki, Higashi-ku, Fukuoka 812-8581, Japan; Electronic address: terasaki@chem.kyushu-univ.jp

"Deceased. buffer gas cooling allows phase space compression, which, in turn, can further increase the number of stored ions. Recently, the depth of a 22-pole trap was measured by analyzing the evaporation rates of trapped ions from a thermodynamical point of view; this result was employed to calculate effective trap depths for several $2 p$-pole ion traps as a function of the $\mathrm{rf}$ amplitude [22,23].

Taking advantage of the high ion density attainable in a multipole ion trap, we have recently been able to apply cavity ring-down spectroscopy directly to the mass-selected ions stored in an octopole trap [3-5]. In the course of these studies, the ions were found to be distributed not uniformly inside the trap, as it was first pointed out in Ref. [3]. In fact, the ion density distribution is not defined by the effective potential alone in a regime where space charge effects become relevant. Understanding these distributions in linear rf traps is extremely important (i) for the optimization of spectroscopic methods that profit from the maximal overlap of laser light with the ion clouds inside the trap, (ii) for the measurement of absolute absorption cross sections, (iii) to accurately estimate the thermalization temperature in reactivity studies, and (iv) for optimization and interpretation of experiments where the ion trap is used as a pick-up cell [24].

The density distribution of a non-neutral plasma in a Penning trap has been extensively studied by Dubin and O'Neil [25]. For a Paul trap, i.e., a quadrupole rf field, profiles of an ion cloud were studied for atomic metal ions and organic molecular ions by monitoring fluorescence and photodissociation of stored ions, respectively [26-28]. These profiles have a Gaussian shape with the maximum ion density at the center of the trap. More recently, even single ions have been observed by fluorescence imaging of Coulomb crystals formed in a linear Paul trap at temperatures around $10 \mathrm{mK}$ [29]. For multipole ion 
traps, on the other hand, only a few measurements have been reported. Walz et al. measured a radial distribution of ions in a three-dimensional hyperbolic octopole ion trap by monitoring fluorescence intensity from stored $\mathrm{Ba}^{+}$ions [30]. They found that Coulomb repulsion between stored ions resulted in two separate ion peaks. Wester and coworkers reported a radial distribution of column densities of $\mathrm{OH}^{-}$ions in a 22-pole ion trap from photodetachment-rate measurements [31]. The distribution showed a rather uniform profile and was explained by a model calculation neglecting Coulomb-repulsion effects; this model was applicable to the measurement performed at a low ion density (less than $10^{3}$ stored ions). They extended the measurement to two-dimensional tomography of the column density [32]. A Coulomb crystal of laser-cooled $\mathrm{Ca}^{+}$ions has recently been formed in a linear octopole ion trap by Okada et al.; the ions were observed by fluorescence imaging for storage of up to $10^{4}$ ions, which is still in the low-density regime [33,34].

In this paper, we report on the measured ion-density profile in a linear octopole ion trap at a high-density regime, where the space charge plays a significant role. Silver dimer cations, $\mathrm{Ag}_{2}^{+}$, are detected via photofragmentation and the twodimensional distributions of column densities are measured as a function of the number of loaded ions and as a function of the amplitude of the rf field. Up to about $10^{9}$ ions are loaded into the trap, which is the space-charge-limit condition for the present ion trap geometry. The distribution profiles are compared with a model calculation, which takes the balance between the trapping force due to the rf field and the Coulomb repulsion among the stored ions into account.

\section{EXPERIMENTAL PROCEDURES}

A schematic diagram of the experimental setup is shown in Fig. 1; a detailed description can be found elsewhere [3]. $\mathrm{Ag}_{2}^{+}$ions were selected by a quadrupole mass filter (labeled QM1, model MAX-4000 by Extrel CMS) from the distribution generated by a magnetron-sputter cluster-ion source. A typical current of the $\mathrm{Ag}_{2}^{+}$ion beam after the mass filter was $200 \mathrm{pA}$, with QM1 operated at relatively low mass resolution $(m / \Delta m \sim 20)$. The selected $\mathrm{Ag}_{2}^{+}$ions were transported by octopole ion guides (IGs) and quadrupole deflectors (QDs) to a linear octopole ion trap.

The ion trap has a length of $40 \mathrm{~cm}$ and an inner diameter of $1.1 \mathrm{~cm}$. The trap is kept at room temperature and is filled with about $0.1 \mathrm{~Pa}$ of $\mathrm{He}$ buffer gas. We assume, thus, that all degrees of freedom of the ions are well thermalized within $0.5 \mathrm{~s}$. The rf potentials at about $3 \mathrm{MHz}$ applied to the octopole's rods were provided by a homemade of generator [35]. The rf amplitude $V_{\mathrm{rf}}$ was varied in the range 95-300 V, while the offset dc potential was kept at $-12 \mathrm{~V}$. The ions were confined in the longitudinal direction using potentials generated by an entrance $\left(\mathrm{L}_{\mathrm{in}}\right)$ and an exit $\left(\mathrm{L}_{\mathrm{out}}\right)$ electrode. The potential of $\mathrm{L}_{\text {in }}$ was switched between -8 and $+5 \mathrm{~V}$ for loading and trapping, respectively. $\mathrm{L}_{\text {out }}$ was held at $+5 \mathrm{~V}$ during loading and trapping and was switched to $-12 \mathrm{~V}$ for extraction of trapped ions. The number $N_{0}$ of ions stored in the trap was measured via monitoring the ion current at the detector (ID1), taking into account the transmission probabilities of IG and
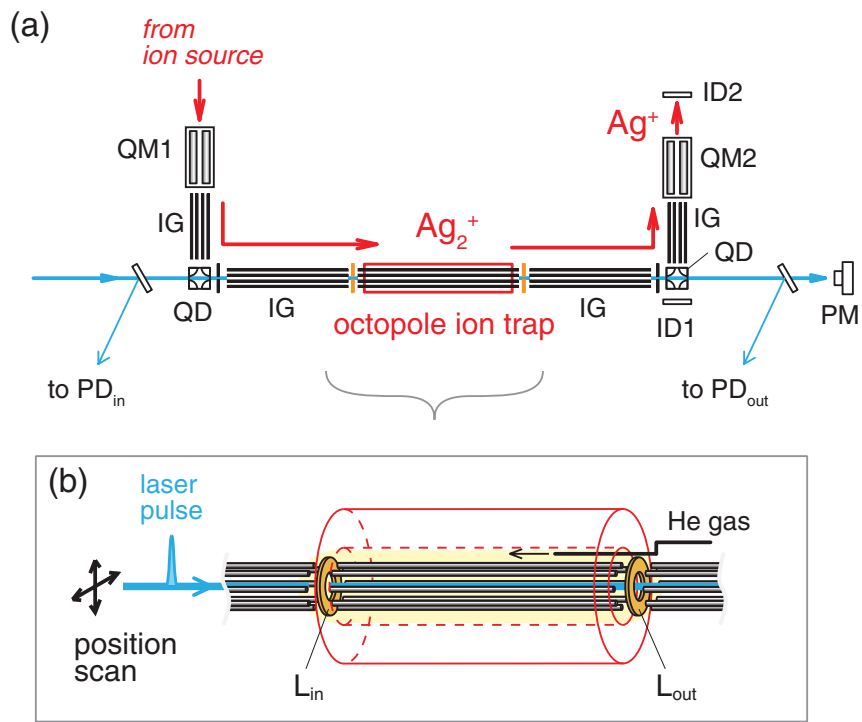

FIG. 1. (Color online) A schematic diagram of the experimental setup. QM1 and QM2, quadrupole mass filters; IGs, octopole ion guides; QDs, quadrupole deflectors; $\mathrm{PD}_{\text {in }}$ and $\mathrm{PD}_{\text {out }}$, photodiodes; PM: a power meter; $L_{\text {in }}$ and $L_{\text {out }}$, entrance and exit electrodes of the ion trap; ID1 and ID2, ion current detectors.

QD. These were estimated to be unity and 0.9 , respectively. The number of stored ions $N_{0}$ was controlled by changing the loading time and/or the ion current. We determined a saturation curve for the loading of the trap as a function of time and from this we determined the maximum loading condition for each $V_{\mathrm{rf}}$.

The column density of the ions was measured by recording the photofragmentation yield introduced by UV laser pulses at $415 \mathrm{~nm}$, near the peak absorption of $\mathrm{Ag}_{2}^{+}$[36]. These were generated by using an optical parametric oscillator (MOPO-HF, Spectra Physics) operated at a repetition rate of $10 \mathrm{~Hz}$, which was attenuated to about $20 \mu \mathrm{J} /$ pulse. With a collimated 2-mm-diameter beam, the intensity was sufficiently low that the photofragment yield depended linearly on the laser pulse energy. The laser beam was aligned carefully to be parallel to the axis of the ion trap. To map the ion density distribution, the laser position was displaced both vertically and horizontally with an interval of $0.5 \mathrm{~mm}$. The laser pulse intensity was monitored during the measurement by the photodiodes $\left(\mathrm{PD}_{\text {in }}\right.$ and $\left.\mathrm{PD}_{\text {out }}\right)$, located before and after the ion trap, outside the vacuum chamber. The signal intensities of the PDs were calibrated by a power meter (PM) placed downstream. The number of photons of the injected laser pulses, $N_{p}$, was determined from the signal intensities of $\mathrm{PD}_{\mathrm{in}}$ measured shot by shot. The intensity ratio between the two PDs was used to confirm that the laser beam was not clipped while scanning its position. The number of photofragment ions, $N_{f}$, was measured by an ion current detector (ID2) after mass analysis using a quadrupole mass filter (QM2). The transmittance of QM2 was estimated to be 0.4 .

The measurement was performed following this procedure: First, $\mathrm{Ag}_{2}^{+}$ions were loaded into the ion trap for a duration between 0.3 and $2.0 \mathrm{~s}$ depending on the number of ions to be stored. Second, the stored ions were thermalized by collisions 
with the He buffer gas at room temperature for $0.5 \mathrm{~s}$. Third, the ions were irradiated with the laser pulse for $1 \mathrm{~s}$, i.e., 10 shots. Finally, the ions were extracted from the trap and the yield of $\mathrm{Ag}^{+}$photofragments was recorded. The above measurement was repeated five times at each laser position.

An absolute value of the local number density of ions, $n(\boldsymbol{r})$, was derived from the relationship:

$$
n(\boldsymbol{r})=\frac{f(\boldsymbol{r})}{\sigma L},
$$

where $\sigma$ is the photodissociation cross section, $L$ is the length of the ion trap, and $f(\boldsymbol{r})$ is the number of fragment ions per photon $\left(N_{f} / N_{p}\right)$. We measured the quantity $f$ as a function of the laser position $r$. Because the trap is $40 \mathrm{~cm}$ long, we assume a uniform distribution of ions along the longitudinal direction of the linear ion trap. The cross section $\sigma$ was determined from the normalization condition:

$$
N_{0}=L \int n(\boldsymbol{r}) d \boldsymbol{r}=\frac{1}{\sigma} \int f(\boldsymbol{r}) d \boldsymbol{r} .
$$

In the present experiments, typical ion densities were found to be of the order of $10^{7}$ ions $/ \mathrm{cm}^{3}$, which corresponds to a Debye length of about $400 \mu \mathrm{m}$.

\section{RESULTS}

Figure 2 shows the result of a two-dimensional scan of an ion cloud containing $5.5 \times 10^{8} \mathrm{Ag}_{2}^{+}$ions. A major part of the trapped ions are found in the outer region rather than at the center of the ion trap. The small variation of the densities depending on the azimuthal angle might be caused by imperfect configuration of the poles and/or other neighboring electrodes [32]. In the following discussion, we assume cylindrically symmetric distributions and will analyze the radial distributions obtained by one-dimensional scans along the horizontal axis. These data are then analyzed using an adiabatic approximation, which provides a rotationally symmetric shape for the effective potential [18].

The radial distributions of the ion density are shown in Fig. 3 for three different amounts of loaded ions, along with model calculations discussed in the following section. For these measurements, $V_{\text {rf }}$ was held constant at $200 \mathrm{~V}$, the optimal value. With $N_{0}=4.0 \times 10^{7}$, the ion distribution is found to

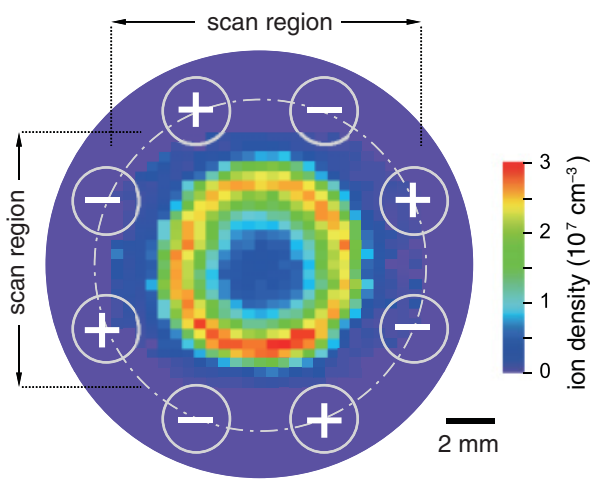

FIG. 2. (Color) Two-dimensional distribution of the $\mathrm{Ag}_{2}^{+}$ion density. The total number $N_{0}$ of stored ions was about $5.5 \times 10^{8}$. The eight circles represent the pole electrodes of the ion trap.

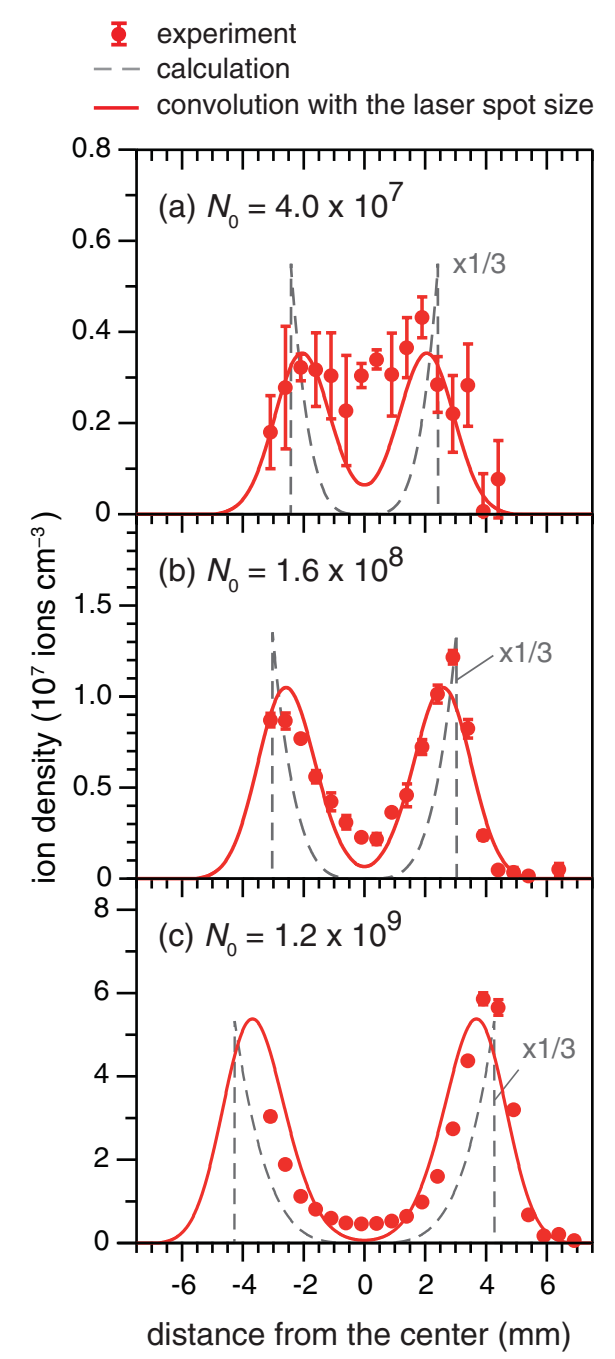

FIG. 3. (Color online) Radial distributions of the ion density obtained by one-dimensional scans on the horizontal axis for different amounts, $N_{0}$, of loaded ions. The amplitude of the rf field $V_{\mathrm{rf}}$ was $200 \mathrm{~V}$. Error bars show statistical standard errors of the measurements. Dashed lines show calculated distributions based on an adiabatic approximation according to Eq. (10) using the measured values for $N_{0}$. Upon convolution of the calculated distribution with a Gaussian function of 2-mm FWHM representing the laser beam profile, we obtained the solid lines, which should be compared with the experimental data. Thermal effects are not considered in these calculations.

be concentrated around the center of the trap [Fig. 3(a)]. As $N_{0}$ is increased, the ion density in the central region increases only slightly while most of the ions are found in the outer region. At the maximum loading condition of $N_{0}=1.2 \times 10^{9}$, the ion density is peaked around $r=4 \mathrm{~mm}$ [Fig. 3(c)]. The fact that the density distribution is confined to the center of the trap when the ion number is low suggests that the ions are well thermalized by collisions with the He buffer gas. Therefore, we interpret the ring profile of the distribution measured for the highest ion density, $N_{0}=1.2 \times 10^{9}$, as the effect of Coulomb repulsion between the trapped ions.

The radial distribution profiles are shown in Fig. 4 for three values of the rf amplitude $V_{\text {rf }}$. Ions were loaded until saturation for each $V_{\mathrm{rf}}$; the number of ions was measured to be 
(a)

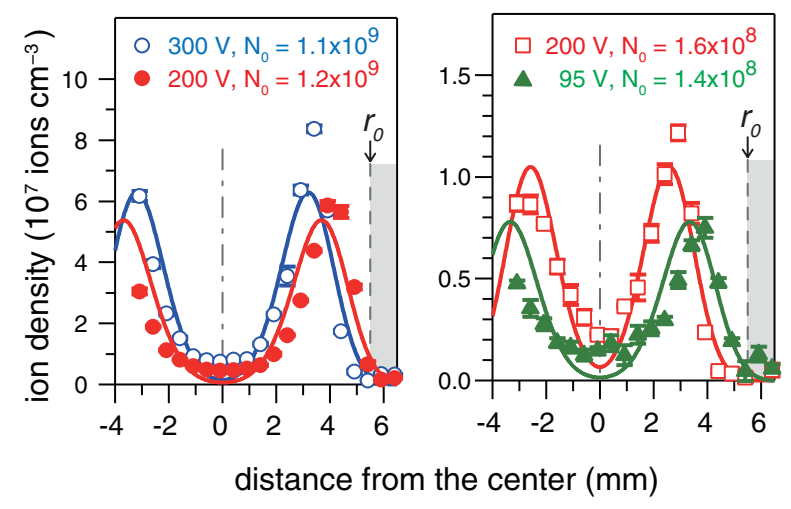

FIG. 4. (Color online) Experimental (symbols) and calculated (line) radial distribution profiles of the ion density for various rf amplitude $V_{\text {rf }}$. (a) The trap is fully loaded with $N_{0}=1.1 \times 10^{9}$ and $1.2 \times 10^{9}$ ions at $V_{\mathrm{rf}}=300$ and $200 \mathrm{~V}$, respectively. (b) The trap is fully loaded with $N_{0}=1.4 \times 10^{8}$ ions at $V_{\mathrm{rf}}=95 \mathrm{~V}$ and partially loaded with $1.6 \times 10^{8}$ ions at $V_{\mathrm{rf}}=200 \mathrm{~V}$ [the latter are the same data as in Fig. 3(b)].

$1.4 \times 10^{8}, 1.2 \times 10^{9}$, and $1.1 \times 10^{9}$ for $V_{\mathrm{rf}}=95,200$, and $300 \mathrm{~V}$, respectively. The number of ions increased by a factor of 8.6 when $V_{\mathrm{rf}}$ was changed from 95 to $200 \mathrm{~V}$, while it slightly decreased when $V_{\text {rf }}$ was changed from 200 to $300 \mathrm{~V}$. Although the total number of ions varied only slightly between $V_{\mathrm{rf}}=200$ and $300 \mathrm{~V}$, the distributions exhibited clearly different profiles; with the higher rf amplitude the density peak became sharper and was shifted by $0.5 \mathrm{~mm}$ toward the center of the trap. In Fig. 4(b), the measurement at $V_{\mathrm{rf}}=200 \mathrm{~V}$ shown in Fig. 3(b), obtained without filling the trap, is superimposed for comparison. We note that, although the total number of ions in Fig. 3(b) and that in the trap filled at $V_{\mathrm{rf}}=95 \mathrm{~V}$ are approximately the same, the distributions are different.

In the above analyses of the absolute ion densities, the photodissociation cross section, $\sigma$, was evaluated by Eq. (2) for each measurement. It was found to be $\sigma=(5 \pm 1) \times$ $10^{-17} \mathrm{~cm}^{2}$ at $415 \mathrm{~nm}$ for the present $300-\mathrm{K}$ ion trap. The uncertainty represents a statistical error of the measurement; we mention that this evaluation may have an additional systematic error due to uncertainties in the estimation of the ion transmittance through the ion optics (see Sec. II) and in the measurement of the laser pulse energy. Note that, as the spectral profile of photoabsorption is dependent on the temperature of the ions, the cross section at a given wavelength changes accordingly [36].

\section{MODEL CALCULATION}

To explain the radial distributions of the ion density dependent on the trapping conditions, we have performed a model calculation based on an adiabatic approximation [18]. Here, effects of the static potential produced by $\mathrm{L}_{\text {in }}$ and $\mathrm{L}_{\text {out }}$ are neglected because they are much smaller than the magnitude of the effective potential for the long trap we have used $(40 \mathrm{~cm})$. Thus, we assume a cylindrical symmetry along the trap axis. The transverse motion of the ions in a multipole ion trap is described as a motion in an effective electric potential [37] expressed by

$$
\Phi_{\mathrm{eff}}(r)=\frac{p^{2}}{4} \frac{q V_{\mathrm{rf}}^{2}}{m \Omega^{2} r_{0}^{2}}\left(\frac{r}{r_{0}}\right)^{2 p-2},
$$

where $q, m, 2 p, \Omega / 2 \pi$, and $r_{0}$ denote the ion charge, the ion mass, the number of poles, the frequency of the rf field, and the inscribed radius of the ion trap, respectively. In general, the equilibrium ion density $n(r)$ in such a potential at a given temperature $T$, is described by

$$
n(r)=n_{0} \exp \left[-\frac{q}{k_{B} T}\left(\Phi_{\mathrm{eff}}(r)+\Phi_{\mathrm{sc}}(r)\right)\right],
$$

where $n_{0}$ and $k_{B}$ are a normalization and Boltzmann's constants, respectively, and $\Phi_{\mathrm{sc}}(r)$ is the electric potential due to the space charge of the ion cloud $[20,30]$. In the general case, Eq. (4) is nonlinear because $\Phi_{\mathrm{sc}}(r)$ is related to the local ion density by Poisson's equation,

$$
\nabla^{2} \Phi_{\mathrm{sc}}(r)=-\frac{q n(r)}{\varepsilon_{0}} .
$$

However, if one assumes the space charge effect to be negligible, Eq. (4) simplifies to

$$
n(r)=n_{0} \exp \left[-\frac{q}{k_{B} T} \Phi_{\text {eff }}(r)\right] .
$$

This approximation was applied to explain an ion distribution in a 22-pole trap containing about $10^{3}$ ions [31]. In the present study, we have about 5 to 6 orders of magnitude more ions so that we load the trap until saturation. Therefore, we cannot neglect the space charge effect, and as a result we rearrange Eq. (4) as

$$
-\Phi_{\mathrm{sc}}(r)=\frac{k_{B} T}{q} \ln \frac{n(r)}{n_{0}}+\Phi_{\mathrm{eff}}(r) .
$$

At zero temperature the space charge due to the ion distribution must exactly counterbalance the effect of the external potential $\Phi_{\text {eff }}$. That is to say, neglecting the energy due to the thermal motion of the ions allows one to recast Eq. (4) as [38]

$$
\Phi_{\text {eff }}(r)+\Phi_{\mathrm{sc}}(r)=0 .
$$

Equations (5) and (8) thus lead to

$$
n(r)=\frac{\varepsilon_{0}}{q} \nabla^{2} \Phi_{\text {eff }}(r),
$$

which determines the radial profile of the ion density. We will see later that this approximation is not entirely fulfilled, but it is nevertheless sufficiently good to explain most of our experimental data. An alternative approach to the general derivation of the charge distribution in a cylindrically symmetrical external potential is described in the Appendix. Using Eq. (3) for the effective potential, we obtain the following radial distribution from Eq. (9):

$$
n(r)=p^{2}(p-1)^{2} \frac{\varepsilon_{0} V_{\mathrm{rf}}^{2}}{m \Omega^{2} r_{0}^{4}}\left(\frac{r}{r_{0}}\right)^{2 p-4} .
$$

At $T=0$, we expect a sharp cut in the distribution at $r_{\max }$, which is determined by the number $N_{0}$ of stored ions. In an octopole ion trap $(p=4)$, the ion density is proportional to $r^{4}$. Note that instead, in the case of a quadrupole $(p=2)$, Eq. (10) 


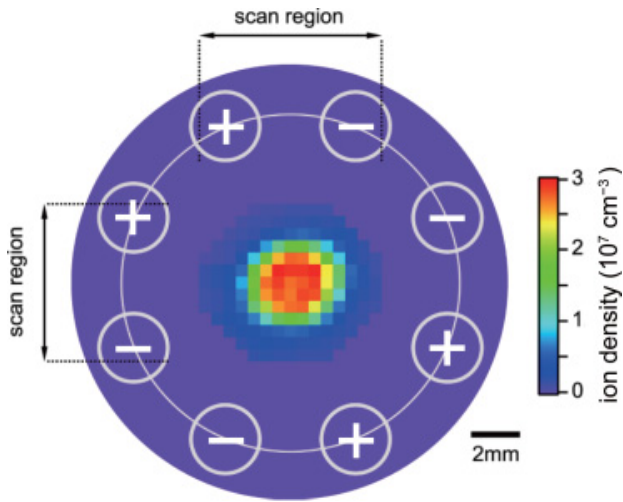

FIG. 5. (Color) A two-dimensional distribution of ion densities measured for $\mathrm{Ag}_{2}^{+}$ions in a quasiquadrupole ion trap, which should be compared with Fig. 2. In this measurement the neighboring poles were wired pairwise together to form four pairs of electrodes. The total number of ions stored was about $1.3 \times 10^{8}$ in this particular condition with $V_{\mathrm{rf}}=360 \mathrm{~V}$. The eight circles represent the pole electrodes of the ion trap, along with the polarity of the rf voltage applied to each electrodes.

predicts a constant ion distribution. In order to compare these predictions with the experimental data, the distribution $n(r)$ was evaluated in the interval $\left[0, r_{\max }\right]$ and then convoluted with a Gaussian function, $2 \mathrm{~mm}$ FWHM, representing the laser-beam diameter. The results of the calculations are shown in Figs. 3 and 4 together with the experimental data. The maximal radius $r_{\max }$ is such that the integral of $n(r)$ over a 40-cm-long cylinder of radius $r_{\max }$ corresponds to the measured $N_{0}$.

The standard way to create multipole potentials is by approximating the hyperbolic equipotential surfaces with cylindrical electrodes. In general, it is always possible to approximate a lower order multipole with the electrodes configuration designed for a higher order one. Thus, we approximated a quadrupole by wiring the neighboring poles of our octopole trap together to form four pairs of electrodes. The ion distribution measured for this quasiquadrupole ion trap is shown in Fig. 5. The ions are concentrated around the axis with a rather flat density profile as predicted by the present model calculation. As the surface of the eight rods cannot perfectly follow the equipotential lines of a quadrupole potential, this quadrupole trap is not perfect and provides a smaller trapping volume than an ordinary four rods configuration.

\section{DISCUSSION}

The distributions calculated by using Eq. (10) are compared with experimental results in Fig. 3. Note that three curves calculated for different $N_{0}$ have the same profile except for $r_{\max }$, which was determined to be $4.3,3.0$, and $2.4 \mathrm{~mm}$ for $N_{0}=1.2 \times 10^{9}, 1.6 \times 10^{8}$, and $4.0 \times 10^{7}$, respectively. The calculated curves reproduce well the overall features of the experimental results, particularly for larger $N_{0}$. This agreement indicates that the ring profiles of the ion distribution are mainly governed by the space charge effect, which forces the stored ions toward the outer region. Note that the largest discrepancies between the model and the experiment are found near the center of the trap and for a low ion density. This is due to the
$T=0$ approximation, which is a poor approximation where $\Phi_{\text {eff }}$ and $\Phi_{\mathrm{sc}}$ are comparable to the energy of the thermal motion, i.e., at small $r$ and/or small $N_{0}$. The room temperature, $k_{B} T=26 \mathrm{meV}$, is equal to $\Phi_{\mathrm{eff}}(r)$ at $r=2.2 \mathrm{~mm}$ for $V_{\mathrm{rf}}=$ $200 \mathrm{~V}$.

Figures 2 and 5 show that the maximal ion densities in an octopole and in a quadrupole are similar, but the latter provides the maximum around the center of the trap, whereas in the former the maximal density is found on a ring at larger radius. Since it is easier to overlap a laser beam to an ion cloud that is concentrated around the center of the trap than to a ring, a quadrupole is probably the most favorable rf linear trap for laser spectroscopy. However, since the phase space acceptance of a quadrupole is lower than that of every other higher order linear rf trap, it is possible that many ions injected into a quadrupole are lost. This makes the loading time of a quadrupole longer than for a higher order multipole. In order to optimize the loading time and the laser beam overlap with the ions, one can imagine using a high-order multipole trap for loading and thermalizing the ions and then switching it to a quadrupole for spectroscopy. This can be done by controlling the potential on each electrode independently.

According to Eq. (10), the maximum radius $r_{\max }$ within which ions are stably stored is determined by the total number of ions and by $V_{\mathrm{rf}}$ - for a given trap geometry and a given $\Omega$. For full traps, however, two other factors reduce the value of $r_{\max }$. One is the physical geometry of the traps: according to Gerlich [18], a typical geometric limit $r_{\text {geom }}$ is $0.8 r_{0}$ due to the space necessary for micromotion wiggling. The other is the breakdown of the adiabatic approximation due to large rf amplitude. The adiabaticity is quantified by means of a dimensionless adiabaticity parameter $\eta(r)$, as described in Refs. [18,39]:

$$
\eta(r)=2 p(p-1) \frac{q V_{\mathrm{rf}}}{m \Omega^{2} r_{0}^{2}}\left(\frac{r}{r_{0}}\right)^{p-2} .
$$

There is a maximum value of $\eta=\eta_{\max }$ for which the rf heating makes the motion of the ions unstable. Since $\eta(r)$ increases with $r$, there is a critical radius $r_{\mathrm{c}}$ at which $\eta\left(r_{\mathrm{c}}\right)=\eta_{\max }$. Whereas $r_{\mathrm{c}}$ decreases with increasing $V_{\mathrm{rf}}, r_{\text {geom }}$ is independent of $V_{\mathrm{rf}}$. Therefore, $r_{\max }$ is equal to $r_{\text {geom }}$ at a sufficiently low $V_{\text {rf }}$. As $V_{\text {rf }}$ grows, the number of stored ions increases according to Eq. (10) until $r_{\mathrm{c}}$ is reduced to $r_{\mathrm{geom}}$. A further increase of $V_{\mathrm{rf}}$ causes a loss of the ions because $r_{\max }$ (now equal to $r_{\mathrm{c}}$ ) is reduced. We summarize these two effects as $r_{\max }=\min \left[r_{\mathrm{c}}, r_{\text {geom }}\right]$. This behavior has been observed by Mikosch et al. [23] in their measurements of the trapping potential depth.

The reduction of the maximum radius $r_{\max }$ observed when $V_{\text {rf }}$ is changed from 200 to $300 \mathrm{~V}$ as shown in Fig. 4 is attributed to the decrease of $r_{\mathrm{c}}$. For the $V_{\mathrm{rf}}=300 \mathrm{~V}$ case, $\eta_{\max }$ is determined to be 0.13 , where the value $r_{\max }=3.7 \mathrm{~mm}$ is obtained from Eq. (10) and from the measured $N_{0}$. We note that this value for $r_{\max }$ is in good agreement with the data shown in Fig. 4. Other groups found $\eta_{\max }=0.36 \pm 0.02$ for a 22-pole trap [23] and $\eta_{\max } \sim 0.2$ for a 3D octopole trap [30]. In addition, Gerlich suggested $\eta_{\max }=0.3$ from numerical simulation [18]. Our value is much lower than the one for the 22-pole trap and the calculated one. The 22-pole experiment 
was done with less than $10^{3}$ ions. Gerlich performed the simulation for a single ion free from perturbation by other ions. In contrast, the present experiment was performed under a strong space-charge effect. The reduction of $\eta_{\max }$ indicates that the ion-ion interaction introduced an additional source of instability.

On the premise that the same $\eta_{\max }=0.13$ be applied for the lower $V_{\mathrm{rf}}$, the values of $r_{\mathrm{c}}$ were calculated to be 4.4 and $6.4 \mathrm{~mm}$ for $V_{\mathrm{rf}}=200$ and $95 \mathrm{~V}$, respectively. Clearly, $r_{\max }$ is limited by $r_{\text {geom }}$ at $V_{\text {rf }}=95 \mathrm{~V}$. At $V_{\text {rf }}=200 \mathrm{~V}$, we were able to store the maximum number of ions and, therefore, $r_{\max }=r_{\mathrm{c}}=r_{\text {geom }}$; the $r_{\mathrm{c}}$ value of $4.4 \mathrm{~mm}$ is in good agreement with the $r_{\max }$ value extracted from Fig. 4 . The value of $r_{\text {geom }} / r_{0}=4.4 / 5.5=0.8$ is consistent with Gerlich's estimation of $r_{\text {geom }} / r_{0} \leqslant 0.8$.

The values of $r_{\text {geom }}$ and $\eta_{\max }$ are specific of this trap geometry and possibly valid only in the high-density limit, but otherwise independent of any other experimental parameter. We have extracted these values using the measured number of ions $N_{0}$ together with Eq. (10), which describes the ion distribution in the high-density limit. As the measurement of absolute numbers is always challenging, it is interesting to consider the inverse problem: namely, the determination of the absolute number of ions in the trap, $N_{0}$, based on a relative measurement of the ion density and on the knowledge of the characteristics of the trap. This is only possible when the densities are high enough for the interaction between ions to become relevant in shaping the ion distribution. Then, one can turn a relative measurement of the ion distribution into a measurement of the interaction strength between ions and, thus, into a measurement of the absolute number of ions. Close to the space charge limit, one obtains $N_{0}$ directly by integration of Eq. (10) between 0 and $r_{\max }^{0}$, where $r_{\max }^{0}$ is the maximum of the ion distribution, which is determined experimentally.

\section{SUMMARY}

We studied the radial distributions of ions stored in a linear octopole ion trap near the space-charge limit by monitoring photofragmentation yields as a function of the laser position. For the highest densities, we observed that the ion distribution has a ring profile. We showed that this is a typical feature of a multipole ion trap. The quadrupole potential, however, is an exception in the family of the linear multipole rf traps as it induces a uniform ion distribution even when the space charge limit is reached. These observations are predicted and explained by a simple model based on equilibrium between the effective potential and that produced by the charge of the ions. The only approximation we used is that the energy related to the thermal motion of the ions inside the trap is negligible in comparison with the effective potential generated by the multipole. This approximation is fulfilled except for the regions where the effective potential is very flat, which, however, contain very few ions when the trap is full.

The maximum adiabaticity parameter, $\eta_{\max }$, was estimated to be 0.13 , when the trap is full. This value is lower than those found in other studies under low ion-density conditions. We tentatively attribute the reduced value of $\eta_{\max }$ to an additional source of instability induced by repulsive forces among the stored ions.
Understanding the space-charge effects allows us to extract the absolute number of ions in the trap and their absolute density based on the relative distribution. This provides a way of measuring absolute numbers without knowledge of absorption cross sections nor detector efficiencies.

\section{ACKNOWLEDGMENTS}

The present study was supported by the Special Cluster Research Project of Genesis Research Institute, Inc.

\section{APPENDIX: CHARGE DISTRIBUTION IN A CYLINDRICALLY SYMMETRICAL EXTERNAL POTENTIAL}

The derivation of Eq. (9) is presented in an alternative way for a general cylindrically symmetrical external potential. The charge distribution is derived under the assumption that it is translational invariant along the $z$ axis, where it extends from negative to positive infinity. Thus, all extensive quantities as charge or energy represent a "per unit length" value in the following.

Assume that a certain amount of charge per unit length, $Q_{0}$, is allowed to distribute freely in a cylindrically symmetrical external potential $\Phi_{\text {ext }}$. In cylindrical coordinates $r, z$, and $\theta$,

$$
\Phi_{\text {ext }}(r, z, \theta)=\Phi_{\text {ext }}(r) .
$$

The charge will distribute radially with density $\rho(r)$ as to minimize the total electrostatic energy $E$ per unit length of $Q_{0}$ in $\Phi_{\text {ext }}$. The density, $\rho(r)$, is to be calculated as follows for any given external potential $\Phi_{\text {ext }}(r)$.

It is convenient to introduce the cumulative charge $Q(r)$, which is the amount of charge per unit length within a cylinder of radius $r$ around the $z$ axis. $Q(r)$ and $\rho(r)$ are related by

$$
\rho(r)=\frac{1}{2 \pi r} Q^{\prime}(r),
$$

where $Q^{\prime}(r)$ is the derivative of $Q(r)$ with respect to $r$, and $Q(r)$ is defined for $0 \leqslant r \leqslant \infty$, its boundary values are $Q(0)=0$ and $Q(\infty)=Q_{0}$. The total energy $E$ per unit length of the charge distribution is composed of the internal energy $E_{\mathrm{i}}$ of $\rho(r)$ [i.e., the electrostatic energy resulting from the repulsion between the volume elements of $\rho(r)]$ and the external energy, $E_{\mathrm{e}}$, of the charge distribution $\rho(r)$ in the external potential. The latter is found by volume-integration of the product $\rho(r) \Phi_{\text {ext }}(r)$ and can be expressed with the help of Eq. (A2) as a one-dimensional integral along the $r$ coordinate:

$$
E_{\mathrm{e}}=\int_{A} \rho(r) \Phi_{\mathrm{ext}}(r) d A=\int_{0}^{\infty} Q^{\prime}(r) \Phi_{\mathrm{ext}}(r) d r,
$$

where $A$ represents the volume in the $r-\theta$ plane. To access $E_{\mathrm{i}}(r)$, it is convenient to derive the strength of the radial internal field $\boldsymbol{E}$ from the first Maxwell equation applied over the surface of an infinite cylinder of radius $r$ along the $z$ axis:

$$
\boldsymbol{E}=\frac{Q(r)}{2 \pi \varepsilon_{0} r} \boldsymbol{e}_{r} .
$$

From classical electrostatics it follows that $E_{\mathrm{i}}$ is given by

$$
E_{\mathrm{i}}=\frac{\varepsilon_{0}}{2} \int_{A} \boldsymbol{E} \cdot \boldsymbol{E} d A=\int_{0}^{\infty} \frac{Q^{2}(r)}{4 \pi \varepsilon_{0} r} d r .
$$


Combining Eqs. (A3) and (A5), the total energy can be written as a single integral over $r$

$$
E=\int_{0}^{\infty}\left\{Q^{\prime}(r) \Phi_{\mathrm{ext}}(r)+\frac{Q^{2}(r)}{4 \pi \varepsilon_{0} r}\right\} d r .
$$

Equation (A6) is already in the canonical form for the calculus of variations. The distribution $Q(r)$ that minimizes $E$ under the given boundary conditions can be found by solving the Euler-Lagrange equation,

$$
\frac{\partial}{\partial r} \frac{\partial L}{\partial Q^{\prime}}-\frac{\partial L}{\partial Q}=0
$$

[1] D. Gerlich, in Low Temperatures and Cold Molecules, edited by I. W. M. Smith (Imperial College Press, London, 2008), p. 121, and references therein.

[2] R. Wester, J. Phys. B 42, 154001 (2009).

[3] A. Terasaki, T. Majima, and T. Kondow, J. Chem. Phys. 127, 231101 (2007).

[4] T. Majima, A. Terasaki, and T. Kondow, Phys. Rev. A 77, 033417 (2008).

[5] A. Terasaki, T. Majima, C. Kasai, and T. Kondow, Eur. Phys. J. D 52, 43 (2009).

[6] K. Hirsch, J. T. Lau, P. Klar, A. Langenberg, J. Probst, J. Rittmann, M. Vogel, V. Zamudio-Bayer, T. Möller, and B. von Issendorff, J. Phys. B 42, 154029 (2009).

[7] O. Kostko, C. Bartels, J. Schwöbel, C. Hock, and B. von Issendorff, J. Phys.: Conf. Ser. 88, 012034 (2007).

[8] K. R. Asmis, M. Brümmer, C. Kaposta, G. Santambrogio, G. von Helden, G. Meijer, K. Rademann, and L. Wöste, Phys. Chem. Chem. Phys. 4, 1101 (2002).

[9] D. J. Goebbert, E. Garand, T. Wende, R. Bergmann, G. Meijer, K. R. Asmis, and D. M. Neumark, J. Phys. Chem. A 113, 7584 (2009).

[10] T. R. Rizzo, J. A. Stearns, and O. V. Boyarkin, Int. Rev. Phys. Chem. 28, 481 (2009)

[11] A. Svendsen, U. J. Lorenz, O. V. Boyarkin, and T. R. Rizzo, Rev. Sci. Instrum. 81, 073107 (2010).

[12] S. Schlemmer, E. Lescop, J. von Richthofen, D. Gerlich, and M. A. Smith, J. Chem. Phys. 117, 2068 (2002).

[13] O. Asvany, P. Kumar, B. Redlich, I. Hegemann, S. Schlemmer, and D. Marx, Science 309, 1219 (2005).

[14] S. Wolf, G. Sommerer, S. Rutz, E. Schreiber, T. Leisner, L. Wöste, and R. S. Berry, Phys. Rev. Lett. 74, 4177 (1995).

[15] W. Paul, B. Lücke, S. Schlemmer, and D. Gerlich, Int. J. Mass. Spectrom. 149, 373 (1995).

[16] W. Paul, S. Schlemmer, B. Lücke, and D. Gerlich, Chem. Phys. 209, 265 (1996).

[17] L. D. Socaciu, J. Hagen, T. M. Bernhardt, L. Wöste, U. Heiz, H. Häkkinen, and U. Landman, J. Am. Chem. Soc. 125, 10437 (2003).

[18] D. Gerlich, Adv. Chem. Phys. 92, 1 (1992).

[19] D. Gerlich, Phys. Scr. T59, 256 (1995). where $L=\left\{Q^{\prime}(r) \Phi_{\text {ext }}(r)+\frac{Q^{2}(r)}{4 \pi \epsilon_{0} r}\right\}$, the integrand of Eq. (A7), is the Lagrangian associated with the variational problem. Equation (A7) leads to

$$
Q(r)=2 \pi \varepsilon_{0} r \Phi_{\mathrm{ext}}^{\prime}(r),
$$

which can be used to finally derive $\rho(r)$ with the help of Eq. (A2):

$$
\frac{\rho(r)}{\varepsilon_{0}}=\left\{\frac{\Phi_{\mathrm{ext}}^{\prime}(r)}{r}+\Phi_{\mathrm{ext}}^{\prime \prime}(r)\right\}=\nabla^{2} \Phi_{\mathrm{ext}}(r),
$$

which is equivalent to Eq. (9) and is applicable to calculate $\rho(r)$ for any given cylindrically symmetric potential $\Phi_{\text {ext }}(r)$.

[20] C. Champenois, J. Phys. B 42, 154002 (2009).

[21] D. Gerlich, in Low Temperatures and Cold Molecules, edited by I. W. M. Smith (Imperial College Press, London, 2008), p. 295, and references therein.

[22] J. Mikosch, U. Frühling, S. Trippel, D. Schwalm, M. Weidemüller, and R. Wester, Phys. Rev. Lett. 98, 223001 (2007)

[23] J. Mikosch, U. Frühling, S. Trippel, R. Otto, P. Hlavenka, D. Schwalm, M. Weidemüller, and R. Wester, Phys. Rev. A 78, 023402 (2008).

[24] F. Bierau, P. Kupser, G. Meijer, and G. von Helden, Phys. Rev. Lett. 105, 133402 (2010).

[25] D. H. E. Dubin and T. M. O’Neil, Rev. Mod. Phys. 71, 87 (1999).

[26] I. Siemers, R. Blatt, T. Sauter, and W. Neuhauser, Phys. Rev. A 38, 5121 (1988).

[27] P. H. Hemberger, N. S. Nogar, J. D. Williams, R. G. Cooks, and J. E. P. Syka, Chem. Phys. Lett. 191, 405 (1992).

[28] C. D. Cleven, R. G. Cooks, A. W. Garrett, N. S. Nogar, and P. H. Hemberger, J. Phys. Chem. 100, 40 (1996).

[29] A. Mortensen, E. Nielsen, T. Matthey, and M. Drewsen, Phys. Rev. Lett. 96, 103001 (2006).

[30] J. Walz, I. Siemers, M. Schubert, W. Neuhauser, R. Blatt, and E. Teloy, Phys. Rev. A 50, 4122 (1994).

[31] S. Trippel, J. Mikosch, R. Berhane, R. Otto, M. Weidemüller, and R. Wester, Phys. Rev. Lett. 97, 193003 (2006).

[32] R. Otto, P. Hlavenka, S. Trippel, J. Mikosch, K. Singer, M. Weidemüller, and R. Wester, J. Phys. B 42, 154007 (2009).

[33] K. Okada, K. Yasuda, T. Takayanagi, M. Wada, H. A. Schuessler, and S. Ohtani, Phys. Rev. A 75, 033409 (2007).

[34] K. Okada, T. Takayanagi, M. Wada, S. Ohtani, and H. A. Schuessler, Phys. Rev. A 80, 043405 (2009).

[35] R. M. Jones and S. L. Anderson, Rev. Sci. Instrum. 71, 4335 (2000).

[36] K. Egashira, C. Bartels, T. Kondow, and A. Terasaki, Eur. Phys. J. D 63, 183 (2011).

[37] Our notation differs from the one used by Gerlich [18], in that we divide the effective mechanical potential by the charge $q$ in order to obtain an effective electric potential.

[38] H. G. Dehmelt, Adv. At. Mol. Phys. 3, 53 (1967).

[39] E. Teloy and D. Gerlich, Chem. Phys. 4, 417 (1974). 